\title{
To the Schwarzschild Solution in General Relativity
}

\author{
Valery V. Vasiliev, Leonid V. Fedorov \\ Institute of Problems in Mechanics, Russian Academy of Sciences, Moscow, Russia \\ Email: vvvas@dol.ru
}

How to cite this paper: Vasiliev, V.V. and Fedorov, L.V. (2018) To the Schwarzschild Solution in General Relativity. Journal of Modern Physics, 9, 2482-2494.

https://doi.org/10.4236/jmp.2018.914160

Received: November 28, 2018

Accepted: December 24, 2018

Published: December 27, 2018

Copyright $\odot 2018$ by authors and Scientific Research Publishing Inc. This work is licensed under the Creative Commons Attribution International License (CC BY 4.0).

http://creativecommons.org/licenses/by/4.0/

\begin{abstract}
The paper is concerned with the history of the spherically symmetric static problem solution of General Relativity found in 1916 by K. Schwarzschild [1] [2] which is interpreted in modern physics as the background of the objects referred to as Black Holes. First, the modern interpretation this solution which does not exactly coincide with original solution obtained by $\mathrm{K}$. Schwarzschild is discussed. Second, the basic equations of the original Schwarzschild solution are presented in modern notations allowing us to compare existing and original solutions. Finally, a modification of the Schwarzschild approach is proposed allowing us to arrive at the exact solution of the Schwarzschild problem.
\end{abstract}

\section{Keywords}

General Relativity, Spherically Symmetric Problem, Schwarzschild's Solution, Black Holes

\section{Spherically Symmetric Static Problem of General Relativity}

Spherically symmetric problem is one of the most discussed problems of General Relativity Theory (GRT) widely described in the literature [3] [4] [5] [6] [7]. This paper is concerned with the analysis of the original Schwarzschild solution of this problem in association with its modern interpretation and possible generalization.

The line element for the spherically symmetric problem is traditionally taken in the following form:

$$
\mathrm{d} s^{2}=g^{2} \mathrm{~d} r^{2}+\rho^{2}\left(\mathrm{~d} \theta^{2}+\sin ^{2} \theta \mathrm{d} \varphi^{2}\right)-h^{2} c^{2} \mathrm{~d} t^{2}
$$

Here $r, \theta, \varphi$ and $t$ are space spherical and time coordinates, $g, \rho, h$ are the 
metric coefficients that depend on the radial coordinate $r$ only. The basic equations of the General Relativity Theory (GRT) link the Einstein tensor $E_{i}^{i}$ with the metric coefficients as [3]

$$
\begin{gathered}
E_{1}^{1}=\frac{1}{\rho^{2}}-\frac{1}{g^{2}} \frac{\rho^{\prime}}{\rho}\left(\frac{\rho^{\prime}}{\rho}+\frac{2 h^{\prime}}{h}\right) \\
E_{2}^{2}=E_{3}^{3}=-\frac{1}{g^{2}}\left[\frac{h^{\prime \prime}}{h}+\frac{\rho^{\prime \prime}}{\rho}+\frac{\rho^{\prime}}{\rho}\left(\frac{h^{\prime}}{h}-\frac{g^{\prime}}{g}\right)-\frac{g^{\prime} h^{\prime}}{g h}\right] \\
E_{4}^{4}=\frac{1}{\rho^{2}}-\frac{1}{g^{2}}\left[\left(\frac{\rho^{\prime}}{\rho}\right)^{2}+\frac{2 \rho^{\prime \prime}}{\rho}-\frac{2 \rho^{\prime} g^{\prime}}{\rho g}\right]
\end{gathered}
$$

where $(\ldots)^{\prime}=\mathrm{d}(\ldots) / \mathrm{d} r$. The components of the Einstein tensor are proportional to the energy tensor $T_{i}^{i}$, i.e.

$$
E_{i}^{i}=\kappa T_{i}^{i}
$$

where

$$
\kappa=8 \pi \gamma / c^{4}
$$

is the GRT gravitational constant depending on the Newton constant $\gamma$ and the velocity of light $c$. Finally, the energy tensor (and the Einstein tensor which is proportional to it) must satisfy the following conservation equation:

$$
\left(T_{1}^{1}\right)^{\prime}-\frac{2 \rho^{\prime}}{\rho}\left(T_{2}^{2}-T_{1}^{1}\right)+\frac{h^{\prime}}{h}\left(T_{1}^{1}-T_{4}^{4}\right)=0
$$

We use mixed components of the tensors $E$ and $T$ because for the problem under study they coincide with the corresponding physical components. The energy tensor depends on the space structure. Particularly, for the empty space

$$
T_{i}^{i}=0,(i=1,2,3,4)
$$

and Equations (2)-(4) are homogeneous. Inside a solid sphere with radius $a$,

$$
T_{1}^{1}=\sigma_{r}, T_{2}^{2}=T_{3}^{3}=\sigma_{\theta}, T_{4}^{4}=\mu c^{2}
$$

where $\sigma_{r}$ and $\sigma_{\theta}$ are the radial and the circumferential stresses and $\mu$ is the material density.

Consider the external space $(r \geq a)$. Taking $E_{4}^{4}=0$, we can reduce Equation (4) to the following form:

$$
\frac{\mathrm{d}}{\mathrm{d} r}\left[\frac{\rho_{e}\left(\rho_{e}^{\prime}\right)^{2}}{g_{e}^{2}}\right]=\rho_{e}^{\prime}
$$

Equation (10) can be readily integrated to give

$$
g_{e}^{2}=\frac{\rho_{e}\left(\rho_{e}^{\prime}\right)^{2}}{\rho_{e}+C_{1}}
$$

where $C_{1}$ is the integration constant and the functions with subscript " $e$ " correspond to the external space. Substituting this result in Equation (2), taking $E_{1}^{1}=0$ and integrating, we get 


$$
h_{e}^{2}=C_{2}\left(1+\frac{C_{1}}{\rho_{e}}\right)
$$

It looks like substituting Equations (11) and (12) into Equation (3) in which $E_{2}^{2}=0$, we can determine the function $\rho_{e}(r)$. But this is not the case-under such substitution, Equation (3) is satisfied identically for any function $\rho_{e}(r)$. This result can be predicted-since the components of the Einstein tensor $E_{i}^{i}$ satisfy Equation (7), Equations (2)-(4) are not mutually independent and any solution of two of them identically satisfy the third equation.

Thus, we have only two Einstein equations for three unknown functions $g(r), \rho(r)$ and $h(r)$. The fact that the set of GTR equations is not complete and must be supplemented with some coordinate conditions was first mentioned by D. Hilbert [8]. By now, the general form of these conditions has not been developed, though some particular forms (e.g., the so-called De-Donder-Fock harmonic coordinate conditions) have been used in spherically symmetric problem [9].

Not knowing the function $\rho_{e}(r)$, we can make some qualitative conclusions about its behavior. It is natural to suppose the for $r \rightarrow \infty$ we should have $\rho_{e} \rightarrow r$ and Equations (11) and (12) should reduce to the corresponding results of the Newton gravitation theory, according to which [10]

$$
g_{\infty}^{2}=1-\frac{2 \varphi}{c^{2}}, h_{\infty}^{2}=1+\frac{2 \varphi}{c^{2}}
$$

where $\varphi=-\gamma m / r$ is the Newton gravitational potential and $m$ is the mass inducing the gravitation field. Equations (13) allows us to determine the constants in Equations (11) and (12) and to present the result as

$$
g_{e}^{2}=\frac{\left(\rho_{e}^{\prime}\right)^{2}}{1-r_{g} / \rho_{e}}, h_{e}^{2}=1-\frac{r_{g}}{\rho_{e}}
$$

Here,

$$
r_{g}=2 m \gamma / c^{2}
$$

is the so-called gravitational radius sometimes referred to as the Schwarzschild radius (though $\mathrm{K}$. Schwarzschild did not use this term).

Consider the internal space $(0 \leq r \leq a)$. For an elastic sphere with known density, we have totally four equations, i.e., Equations (2)-(4) in which the left-hand parts are specified by Equations (5) and (9) and Equation (7) in which the energy tensor should be expressed with the aid of Equation (9). These equations include five unknown functions-the metric coefficients $g, \rho, h$ and the stresses $\sigma_{r}$ and $\sigma_{\theta}$. To solve the problem, we must supplement the GRT equations with the equations for stresses similar to the compatibility equations of the Theory of Elasticity. Such equations can be derived [11], but we restrict ourselves to a particular solution obtained by K. Schwarzschild for a sphere consisting of a perfect incompressible fluid. In this case, $\sigma_{r}=\sigma_{\theta}=-p(r)$ in which $p$ is the pressure in the fluid and the fluid density $\mu_{0}$ does not depend on $p$ and $r$. 
Taking $E_{4}^{4}=\kappa \mu_{0} c^{2}$ in Equation (4), we can reduce it to the form [12]

$$
\frac{\mathrm{d}}{\mathrm{d} r}\left[\frac{\rho_{i}}{g_{i}^{2}}\left(\rho_{i}^{\prime}\right)\right]=\left(1-\kappa \mu_{0} c^{2} \rho_{i}^{2}\right) \rho_{i}^{\prime}
$$

Equation (16) can be readily integrated to give

$$
g_{i}^{2}=\frac{\left(\rho^{\prime}\right)^{2}}{1-\lambda^{2} \rho_{i}^{2}-C_{3} / \rho_{i}}
$$

where

$$
\lambda^{2}=\frac{1}{3} \kappa \mu_{0} c^{2}
$$

and the functions with subscript " $i$ " correspond to the internal space. We do not know the function $\rho_{i}(r)$, but can make some reasonable prediction concerning its behavior. Particularly, it is natural to suppose that at the sphere center $\rho_{i}=r=0$. Then, in accordance with the symmetry condition at the sphere center, we should take $C_{3}=0$ and Equation (17) becomes

$$
g_{i}^{2}=\frac{\left(\rho_{i}^{\prime}\right)^{2}}{1-\lambda^{2} \rho_{i}^{2}}
$$

Continue the derivation and consider Equations (2) and (7). Taking $E_{1}^{1}=-\kappa p$ and $T_{1}^{1}=T_{2}^{2}=-p, T_{4}^{4}=\mu_{0} c^{2}$, we arrive at

$$
\frac{1}{\rho_{i}^{2}}-\frac{\rho_{i}^{\prime}}{g_{i}^{2} \rho_{i}}\left(\frac{\rho_{i}^{\prime}}{\rho_{i}}-\frac{2 h_{i}^{\prime}}{h_{i}}\right)=-\kappa p, p^{\prime}+\frac{h_{i}^{\prime}}{h}\left(p+\mu_{0} c^{2}\right)=0
$$

Consider the first Equation. Substituting Equation (19), express the time metric coefficient $h_{i}$, i.e.,

$$
\frac{\mathrm{d} h_{i}}{h_{i}}=\frac{\mathrm{d} \rho_{i}}{2}\left[\frac{\rho_{i}}{1-\lambda^{2} \rho_{i}^{2}}\left(\kappa p+\frac{1}{\rho_{i}^{2}}\right)-\frac{1}{\rho_{i}}\right]
$$

and rewrite the second equation of Equations (20) as

$$
\mathrm{d} p+\frac{\mathrm{d} h_{i}}{h_{i}}\left(p+\mu_{0} c^{2}\right)=0
$$

Substituting Equation (21) and using Equation (18), we arrive at the following equation for the pressure:

$$
\mathrm{d} p+\frac{\lambda^{2} \rho_{i} \mathrm{~d} \rho_{i}}{2\left(1-\lambda^{2} \rho_{i}^{2}\right)}\left(p+\mu_{0} c^{2}\right)\left(1+\frac{3 p}{\mu_{0} c^{2}}\right)=0
$$

The solution of Equation (23) that satisfies the boundary condition on the free sphere surface $r=a$ according to which $p\left(\rho_{i}=\rho_{a}\right)=0$ is

$$
p=-\mu_{0} c^{2} \frac{\sqrt{1-\lambda^{2} \rho_{i}^{2}}-\sqrt{1-\lambda^{2} \rho_{a}^{2}}}{\sqrt{1-\lambda^{2} \rho_{i}^{2}}-3 \sqrt{1-\lambda^{2} \rho_{a}^{2}}}
$$

where $\rho_{a}=\rho_{i}(r=a)=\rho_{e}(r=a)$. To determine $h_{i}$, integrate Equation (22) to get $h_{i}\left(p+\mu_{0} c^{2}\right)=C_{4}$ in which $C_{4}$ is the integration constant that can be 
found from the boundary condition on the sphere surface $r=a$ according to which $h_{e}\left(\rho_{e}=\rho_{a}\right)=h_{i}\left(\rho_{i}=\rho_{a}\right)$. Using the second equation in Equations (14), we finally have

$$
h_{i}=\frac{\sqrt{1-r_{g} / \rho_{a}}}{2 \sqrt{1-\lambda^{2} \rho_{a}^{2}}}\left(3 \sqrt{1-\lambda^{2} \rho_{0}^{2}}-\sqrt{1-\lambda^{2} \rho_{i}^{2}}\right)
$$

It should be noted that substitution of the obtained solutions $g_{i}\left(\rho_{i}\right), h_{i}\left(\rho_{i}\right)$ and $p\left(\rho_{i}\right)$ in the Einstein Equation (3) where $E_{2}^{2}=-\kappa p$, does not allow us to find the function $\rho_{i}(r)$ because Equation (3) is identically satisfied for any function $\rho_{i}(r)$. The situation is similar to the external space and for the same reason-since the right-hand parts of Equations (2) and (3) satisfy Equation (7), only three of Equations (2)-(4) and (7) are mutually independent.

Thus, Equation (1) which specifies the metric forms of the external and internal spaces of the fluid sphere can be presented as

$$
\begin{aligned}
\mathrm{d} s_{e}^{2}= & \frac{\left(\rho_{e}^{\prime}\right)^{2} \mathrm{~d} r^{2}}{1-r_{g} / \rho_{e}}+\rho_{e}^{2}\left(\mathrm{~d} \theta^{2}+\sin ^{2} \theta \mathrm{d} \varphi^{2}\right)-\left(1-\frac{r_{g}}{\rho_{e}}\right) c^{2} \mathrm{~d} t^{2} \\
\mathrm{~d} s_{i}^{2}= & \frac{\left(\rho_{i}^{2}\right) \mathrm{d} r^{2}}{1-\lambda^{2} \rho_{i}^{2}}+\rho_{i}^{2}\left(\mathrm{~d} \theta^{2}+\sin ^{2} \theta \mathrm{d} \varphi^{2}\right) \\
& -\frac{1-r_{g} / \rho_{a}}{4\left(1-\lambda^{2} \rho_{a}^{2}\right)}\left(3 \sqrt{1-\lambda^{2} \rho_{a}^{2}}-\sqrt{1-\lambda^{2} \rho_{i}^{2}}\right)^{2} c^{2} \mathrm{~d} t^{2}
\end{aligned}
$$

To fulfill the solution, we need to find two functions $\rho_{e}(r)$ and $\rho_{i}(r)$ such that allow us to satisfy the boundary conditions at the sphere surface $r=a$, i.e.,

$$
\rho_{e}(a)=\rho_{i}(a), g_{e}(a)=g_{i}(a)
$$

However, the equations allowing us to determine these functions are missing in GRT. The same problem exists in the general case-as known, the set of Einstein equations is not complete. In the four-dimensional Riemannian space, this set consists of 10 equations

$$
E^{i j}=-\kappa T^{i j}
$$

for ten components of the metric tensor $g^{i j}$. However the Einstein tensor satisfies equations which are analogous to Equations (7). As a result, only six of Equations (29) are mutually independent and to determine the metric tensor we should supplement Equations (29) with four coordinate conditions for $g^{i j}$. Some authors declare that these conditions cannot be covariant because there forms depend on the particular coordinate frame [3] [8]. Consider some particular cases.

\section{Modern Interpretation of the Schwarzschild Solution}

Traditional description of the Schwarzschild solution can be found elsewhere [3]. The coordinate condition mentioned in the closure of the previous section is taken in the form

$$
\rho_{e}(r)=\rho_{i}(r)=r
$$


though K. Schwarzschild did not use directly this relationship. Applying Equations (30) to Equations (14) and (19), we can specify the metric coefficients for this case and present the metric form in Equation (1) as

$$
\begin{aligned}
\mathrm{d} s_{e}^{2}= & \frac{\mathrm{d} r^{2}}{1-r_{g} / r}+r^{2}\left(\mathrm{~d} \theta^{2}+\sin ^{2} \theta \mathrm{d} \varphi^{2}\right)-\left(1-\frac{r_{g}}{r}\right) c^{2} \mathrm{~d} t^{2} \\
\mathrm{~d} s_{i}^{2}= & \frac{\mathrm{d} r^{2}}{1-\lambda^{2} r^{2}}+r^{2}\left(\mathrm{~d} \theta^{2}+\sin ^{2} \theta \mathrm{d} \varphi^{2}\right) \\
& -\frac{1-r_{g} / a}{4\left(1-\lambda^{2} a^{2}\right)}\left(3 \sqrt{1-\lambda^{2} a^{2}}-\sqrt{1-\lambda^{2} \rho_{i}^{2}}\right)^{2} c^{2} \mathrm{~d} t^{2}
\end{aligned}
$$

To fulfill the solution, we need to satisfy the boundary conditions (28) on the sphere surface $r=a$. The first condition is satisfied automatically, whereas the second one yields

$$
r_{g}=\lambda^{2} a^{3}
$$

However, the parameters $r_{g}$ and $\lambda^{2}$ are specified by Equations (15) and (18) and are known. So, Equation (33) cannot be satisfied in the general case and the second boundary condition in Equations (28) is violated. Substituting formally Equations (15) and (18) in Equation (33), we arrive at the following expression:

$$
m=m_{e}=\frac{4}{3} \pi \mu_{0} a^{3}
$$

which specifies the mass of a homogeneous solid sphere in the Euclidean space. However, the space in GRT is not Euclidean and the mass of the sphere with the metric coefficients corresponding to Equations (19) is

$$
\begin{aligned}
m & =4 \pi \mu_{0} \int_{0}^{a} g_{i} r^{2} \mathrm{~d} r=\frac{2}{\lambda^{2}} \pi a \mu_{0}\left(\frac{1}{\lambda a} \sin ^{-1} \lambda a-\sqrt{1-\lambda^{2} a^{2}}\right) \\
& \approx m_{e}\left(1+\frac{15}{128} \lambda^{2} a^{2}+\cdots\right)
\end{aligned}
$$

As can be seen, the obtained result does not coincide with Equation (34) and the second boundary condition in Equations (28) is not satisfied. The reason for this discrepancy is associated with Equations (30). Equation (4), being originally of the second order, under transformation in accordance with Equation (30) reduces to the equation of the first order. As a result, the solution does not contain the proper number of integration constants allowing us to satisfy the complete set of the boundary conditions.

Thus, the coordinate conditions in Equations (30) do not look suitable for the problem under study.

\section{Original Schwarzschild's Solution}

In 1916 K. Schwarzschild presented the solution of the external spherically symmetric problem [1]. He did not use the final version of the Einstein equations, however the field equations that he applied can be now associated with 
Equations (2)-(4). We can suppose that he understood that only two of these equations were mutually independent because he attracted for the analysis only two equations, i.e. Equations (2) and (4), and ignored Equation (3). The third equation which is necessary to solve the problem, was obtained under the following condition imposed on the determinant of the metric tensor:

$$
\left|g_{m n}\right|=1
$$

Introducing this equation, K. Schwarzschild followed A. Einstein who used it in general theory to specify the coordinate frame [13]. Governing equations of GRT contain symbols $\Gamma_{i j}^{k}$ which include derivatives of the determinant and become zero under condition (33) thus simplifying the equations. However, Equation (33) cannot be directly applied in spherical coordinates in which the volume element in the three-dimensional Euclidean space is $\mathrm{d} v=r^{2} \sin \theta \mathrm{d} r \mathrm{~d} \theta \mathrm{d} \varphi$. To overcome this problem, K. Schwarzschild introduced new variables $x_{i}$ such that

$$
x_{1}=r^{3} / 3, x_{2}=-\cos \theta, x_{3}=\varphi, x_{4}=t
$$

In new coordinates, the volume element becomes $\mathrm{d} v=\mathrm{d} x_{1} \mathrm{~d} x_{2} \mathrm{~d} x_{3}$ and the line element takes the form

$$
\mathrm{d} s^{2}=f_{1} \mathrm{~d} x_{1}^{2}+f_{2}\left[\frac{\mathrm{d} x_{2}^{2}}{1-x_{2}^{2}}+\left(1-x_{2}^{2}\right) \mathrm{d} x_{3}^{2}\right]-f_{4} \mathrm{~d} x_{4}^{2}
$$

Three functions $f_{1}, f_{2}, f_{4}$ can be found from Equations (2) and (4) supplemented with equation $f_{1} f_{2}^{2} f_{4}=1$ which follows from Equation (36). The final solution is

$$
f_{1}=\frac{\left(3 x_{1}+\beta^{3}\right)^{-4 / 3}}{1-\alpha\left(3 x_{1}+\beta^{3}\right)^{-1 / 3}}, \quad f_{2}=\left(3 x_{1}+\beta^{3}\right)^{2 / 3}, \quad f_{4}=1-\alpha\left(3 x_{1}+\beta^{3}\right)^{-1 / 3}
$$

in which $\alpha$ and $\beta^{3}$ are the integration constants. As stated in the Schwarzschild paper, this solution identically satisfies Equation (3) (which should be the case).

The final part of the paper can hardly be understood. Directly following K. Schwarzschild, consider the function $f_{1}$ which can be a source of singularity. Equating the denominator to zero and using Equation (37) for $x_{1}$ we get

$$
\alpha\left(r^{3}+\beta^{3}\right)^{-1 / 3}=1
$$

Let singularity take place at the origin $r=0$. Then, Equation (40) yields $\beta=\alpha$. Introducing the new variable

$$
R=\left(r^{3}+\alpha^{3}\right)^{1 / 3}
$$

and using Equations (38) and (39), K. Schwarzschild arrived at the following final result of his paper:

$$
\mathrm{d} s^{2}=\frac{\mathrm{d} R^{2}}{1-\alpha / R}+R^{2}\left(\mathrm{~d} \theta^{2}+\sin ^{2} \theta \mathrm{d} \varphi^{2}\right)-(1-\alpha / R) c^{2} \mathrm{~d} t^{2}
$$


This form formally coincides with Equation (31), but it should be taken into account that $R$ is not the radial coordinate $r$. The constant $\alpha$ is declared to depend on the mass located at the origin, but is not found.

As can be seen, the first term in Equation (42) becomes singular if $R=\alpha$ or $r=0$. Thus, the original Schwarzschild solution has only one singular point$r=0$.

However, it looks like Equation (42) is not correct. To show this, change $R$ to $r$ in Equation (42) with the aid of Equation (41) to get

$$
\begin{aligned}
\mathrm{d} s^{2}= & \frac{\left(r^{3}+\alpha^{3}\right)^{-4 / 3} r^{4} \mathrm{~d} r^{2}}{1-\alpha\left(r^{3}+\alpha^{3}\right)^{-1 / 3}}+\left(r^{3}+\alpha^{3}\right)^{2 / 3}\left(\mathrm{~d} \theta^{2}+\sin ^{2} \theta \mathrm{d} \varphi^{2}\right) \\
& -\left[1-\alpha\left(r^{3}+\alpha^{3}\right)^{-1 / 3}\right] c^{2} \mathrm{~d} t^{2}
\end{aligned}
$$

As can be proved, the first term of this equation becomes zero at $r=0$ which cannot be true. The origin of the mistake is in Equation (40) from which it follows that $\beta=\alpha$.

To demonstrate the alternative approach, substitute Equations (39) in Equation (38) and return to spherical coordinates with the aid of Equations (37). The resulting equation is

$$
\begin{aligned}
\mathrm{d} s^{2}= & \frac{\left(r^{3}+\beta^{3}\right)^{-4 / 3} r^{4} d r^{2}}{1-\alpha\left(r^{3}+\beta^{3}\right)^{-1 / 3}}+\left(r^{3}+\beta^{3}\right)^{2 / 3}\left(\mathrm{~d} \theta^{2}+\sin ^{2} \theta \mathrm{d} \varphi^{2}\right) \\
& -\left[1-\alpha\left(r^{3}+\beta^{3}\right)^{-1 / 3}\right] c^{2} \mathrm{~d} t^{2}
\end{aligned}
$$

As can be seen, the first term has the proper behavior at $r=0$ if we take $\beta=0$ (not $\beta=\alpha$ as in the Schwarzschild solution). The resulting expression

$$
\mathrm{d} s^{2}=\frac{\mathrm{d} r^{2}}{1-\alpha / r}+r^{2}\left(\mathrm{~d} \theta^{2}+\sin ^{2} \theta \mathrm{d} \varphi^{2}\right)-\left(1-\frac{\alpha}{r}\right) c^{2} \mathrm{~d} t^{2}
$$

completely corresponds to the modern interpretation of the Schwarzschild solution in Equation (31), if we apply the asymptotic Equations (13) which give $\alpha=r_{g}$.

Consider the solution of the internal problem that was published by $\mathrm{K}$. Schwarzschild in 1916 [2]. This solution was not supported by A. Einstein [14] because the concept of an incompressible fluid involves infinitely high velocity of the wave in the fluid which does not correspond to the basic GRT concept. However, the solution for compressible fluids does not demonstrate qualitative deviation from the Schwarzschild solution [15] which is discussed below

The method of the solution is the same that for the external problem, i.e., the new variables in Equations (37) are introduced and the field equations are supplemented with Equation (36). Further, another variable $\chi$ is introduced in accordance with the following equation:

$$
\sin \chi=\lambda\left(r^{3}+\eta^{3}\right)^{1 / 3}
$$


where $\lambda$ is specified by Equation (18) (in the original equation $c=1$ ) and $\eta^{3}$ is the integration constant. The final original result for the space part of the metric form is

$$
\mathrm{d} s_{i}^{2}=\frac{1}{\lambda}\left[\mathrm{d} \chi^{2}+\sin ^{2} \chi\left(\mathrm{d} \theta^{2}+\sin ^{2} \theta \mathrm{d} \varphi^{2}\right)\right]-\left(\frac{3}{2} \cos \chi_{a}-\frac{1}{2} \cos \chi\right)^{2} c^{2} \mathrm{~d} t^{2}
$$

where $\chi_{a}=\chi(r=a)$.

To discuss the result obtained by K. Schwarzschild, change $\chi$ to $r$ using Equation (46). Then, Equation (47) becomes

$$
\begin{aligned}
\mathrm{d} s_{i}^{2}= & \frac{\left(r^{3}+\eta^{3}\right)^{-4 / 3} r^{4} \mathrm{~d} r^{2}}{1-\lambda^{2}\left(r^{3}+\eta^{3}\right)^{2 / 3}}+\left(r^{3}+\eta^{3}\right)^{2 / 3}\left(\mathrm{~d} \theta^{2}+\sin ^{2} \theta \mathrm{d} \varphi^{2}\right) \\
& -\frac{1}{4}\left[3 \sqrt{1-\lambda^{2}\left(a^{3}+\eta^{3}\right)^{2 / 3}}-\sqrt{1-\lambda^{2}\left(r^{3}+\eta^{3}\right)^{2 / 3}}\right]^{2} c^{2} \mathrm{~d} t^{2}
\end{aligned}
$$

The first coefficient becomes zero at $r=0$, which cannot be true. To obtain the realistic metric, we must take $\eta=0$ and arrive at the expression

$$
\mathrm{d} s_{i}^{2}=\frac{\mathrm{d} r^{2}}{1-\lambda^{2} r^{2}}+r^{2}\left(\mathrm{~d} \theta^{2}+\sin ^{2} \theta \mathrm{d} \varphi^{2}\right)-\frac{1}{4}\left(3 \sqrt{1-\lambda^{2} a^{2}}-\sqrt{1-\lambda^{2} r^{2}}\right)^{2} c^{2} \mathrm{~d} t^{2}
$$

In the closure of his paper, K. Schwarzschild analyzed the obtained solution. Particularly, the sphere mass was found in the form

$$
m=\frac{6 \pi}{\kappa \lambda}\left(\chi_{a}-\frac{1}{2} \sin 2 \chi_{a}\right)
$$

which coincides with Equation (35) after transformation with the aid of Equation (46). The first term in Equation (48) becomes singular at some critical sphere radius $a_{g}=1 / \lambda$. This radius coincides with the gravitational radius $r_{g}$ only under the condition imposed by Equation (33). If this equation is valid, the metric form in Equation (48) coincides with Equation (32). But then, the sphere mass is specified by Equation (34) which corresponds to the Euclidean space. However, the mass found by K. Schwarzschild is given by Equation (49) and corresponds to the Riemannian space. Thus, the radius $r_{g}$ cannot be called the Schwarzschild radius.

Now return to Equation (44) which specifies the Schwarzschild solution for the external space. Taking $r \rightarrow \infty$, and performing asymptotic analysis, we can prove that the metric coefficients in Equation (44) reduce to Equations (13) if we take $\alpha=r_{g}$. Then Equation (44) becomes

$$
\begin{aligned}
\mathrm{d} s^{2}= & \frac{\left(r^{3}+\beta^{3}\right)^{-4 / 3} r^{4} \mathrm{~d} r^{2}}{1-r_{g}\left(r^{3}+\beta^{3}\right)^{-1 / 3}}+\left(r^{3}+\beta^{3}\right)^{2 / 3}\left(\mathrm{~d} \theta^{2}+\sin ^{2} \theta \mathrm{d} \varphi^{2}\right) \\
& -\left[1-r_{g}\left(r^{3}+\beta^{3}\right)^{-1 / 3}\right] c^{2} \mathrm{~d} t^{2}
\end{aligned}
$$

In contrast to the traditional Equation (31), this equation contains one more integration constant $-\beta$. This result looks natural because K. Schwarzschild did 
not use Equations (30) and, hence, did not reduce the order of Equation (4). Considering the space with a point mass, we took $\beta=0$ because of the behavior of the first coefficient in Equation (50) at $r=0$ and reduced Equation (44) to Equation (45). But now we study the external space of a fluid sphere for which $r \geq a$. So, we can try to use this constant to obtain the continuous solution in Equations (48) and (50) at the sphere surface. However, matching equations (48) and (50), we can conclude that the second terms can be continuous only if $\beta=0$. Thus, the final form of Equation (50) for the external space of a fluid sphere

$$
\mathrm{d} s^{2}=\frac{\mathrm{d} r^{2}}{1-r_{g} / r}+r^{2}\left(\mathrm{~d} \theta^{2}+\sin ^{2} \theta \mathrm{d} \varphi^{2}\right)-\left(1-\frac{r_{g}}{r}\right) c^{2} \mathrm{~d} t^{2}
$$

coincides with the traditional Equation (31). The first and the third coefficients of Equations (48) and (51) are continuous at $r=a$ if $r_{g}=\lambda^{2} a^{3}$ which coincides with Equation (33). Thus, Equation (36) applied by K. Schwarzschild is not the proper coordinate condition and actually gives the same results that the conditions in Equations (30). The original Schwarzschild solution, as well as it modern interpretation, does not provide the solution which satisfies GRT equations and all asymptotic and boundary conditions for a fluid sphere.

\section{New Model of Space and Spherically Symmetric Problem}

Traditionally GRT is associated with Riemannian geometry which describes the so-called curved space. A three-dimensional curved space can be hardly imagined. This space can be formally embedded into traditional Euclidean space. However such space has six dimensions which do not provide better understanding of the problem. The proposed interpretation of the Riemannian space is based on the following assumptions. First, we assume that the space is not an object of geometry, but is a material substance (ether, physical vacuum or whatever else). Second, we think that the curved space does not exist in reality and the Riemannian geometry is only a mathematical model of a special Euclidean space. This space is not homogeneous and is characterized with so-called space density that is a function of the coordinates to which the space is referred. The space density $d=\mathrm{d} v_{R} / \mathrm{d} v_{E}$ is the ratio of the three-dimensional volume elements corresponding to the Riemannian and to the Euclidean geometries in the same coordinate frame [16] [17]. For spherical coordinates and the metric form in Equation (1), we have $\mathrm{d} v_{R}=g \rho^{2} \sin \theta \mathrm{d} r \mathrm{~d} \theta \mathrm{d} \varphi$ and $\mathrm{d} v_{E}=r^{2} \sin \theta \mathrm{d} r \mathrm{~d} \theta \mathrm{d} \varphi$, so that $d=g(\rho / r)^{2}$. Using Equations (14) and (19), we get for the external and internal spaces

$$
d_{e}=\frac{\rho_{e}^{2} g_{e}}{r^{2}}=\frac{\rho_{e}^{\prime} \rho_{e}^{2}}{r^{2} \sqrt{1-r_{g} / \rho_{e}}}, d_{i}=\frac{\rho_{i}^{2} g_{i}}{r^{2}}=\frac{\rho_{i}^{\prime} \rho_{i}^{2}}{r^{2} \sqrt{1-\lambda^{2} \rho_{i}^{2}}}
$$

The space densities in Equations (52) are characterized with some specific properties. Consider the total density of the external and internal spaces for the fluid sphere with radius a 


$$
\begin{gathered}
D_{e}=4 \pi \int_{a}^{\infty} d_{e} r^{2} \mathrm{~d} r=4 \pi \int_{a}^{\infty} F\left(\rho_{e}, \rho_{e}^{\prime}\right) \mathrm{d} r, F\left(\rho_{e}, \rho_{e}^{\prime}\right)=\frac{\rho_{e}^{\prime} \rho_{e}^{2}}{\sqrt{1-r_{g} / \rho_{e}}} \\
D_{i}=4 \pi \int_{0}^{a} d_{i} r^{2} \mathrm{~d} r=4 \pi \int_{0}^{a} F\left(\rho_{i}, \rho_{i}^{\prime}\right) \mathrm{d} r, F\left(\rho_{i}, \rho_{i}^{\prime}\right)=\frac{\rho_{i}^{\prime} \rho_{i}^{2}}{\sqrt{1-\lambda^{2} \rho_{i}^{2}}}
\end{gathered}
$$

Consider the variational equations providing the minimum values of the functional in Equations (53) and (54), i.e.,

$$
\frac{\partial F}{\partial \rho}-\frac{\partial}{\partial r} \frac{\partial F}{\partial \rho^{\prime}}=0
$$

As can be readily proved, Equation (55) is satisfied identically for both functions $F$ in Equations (53) and (54). Thus, the space densities in Equations (52) provide the minimum total density of the space. However, the space density is caused by gravitation and is minimum in the absence of gravitation, i.e., if the space geometry is Euclidean or if $d=1$ which means that the space tends to become homogeneous with respect to the space density $d$. The condition $d=1$ looks slightly similar to Equation (36) applied by K. Schwarzschild. It has a simple physical and geometrical meaning-gravitation, changing the space geometry, does not affect the space volume.

Taking $d_{e}=1$ and $d_{i}=1$ in Equations (52), we arrive at two differential equations for functions $\rho_{e}(r)$ and $\rho_{i}(r)$, i.e.,

$$
\rho_{e}^{\prime} \rho_{e}^{2}=r^{2} \sqrt{1-r_{g} / \rho_{e}}, \quad \rho_{i}^{\prime} \rho_{i}^{2}=r^{2} \sqrt{1-\lambda^{2} \rho_{i}^{2}}
$$

Consider the second equation. Since $d_{i}=g_{i} \rho_{i}^{2} / r^{2}=1$, the sphere mass becomes

$$
m=4 \pi \mu_{0} \int_{0}^{a} g_{i} \rho_{i}^{2} \mathrm{~d} r=4 \pi \mu_{0} \int_{0}^{a} r^{2} \mathrm{~d} r=\frac{4}{3} \pi \mu_{0} a^{3}
$$

and coincides with the Euclidean mass in Equation (34) which means that the condition in Equation (33) is valid and $\lambda^{2}=r_{g} / a^{3}$. Then, the second equation in Equations (56) reduces to

$$
\rho_{i}^{\prime} \rho_{i}^{2}=r^{2} \sqrt{1-r_{g} \rho_{i}^{2} / a^{3}}
$$

The solution of Equation (57) which satisfies the boundary condition $\rho_{i}(r=0)=0$ is [12]

$$
\frac{1}{\sqrt{\bar{r}_{g}}} \sin ^{-1}\left(\bar{\rho}_{i} \sqrt{\bar{r}_{g}}\right)-\bar{\rho}_{i} \sqrt{1-\bar{r}_{g} \bar{\rho}_{i}^{2}}=\frac{2}{3} \bar{r}_{g} \bar{r}^{3}
$$

where

$$
\bar{r}=r / a, \bar{\rho}=\rho / a
$$

Recall that at the sphere surface $\rho_{i}(r=a)=\rho_{a}$. Taking $\bar{r}=1$ and $\bar{\rho}_{i}=\bar{\rho}_{a}$ in Equation (58), we get

$$
\frac{1}{\sqrt{\bar{r}_{g}}} \sin ^{-1}\left(\bar{\rho}_{a} \sqrt{\bar{r}_{g}}\right)-\bar{\rho}_{a} \sqrt{1-\bar{r}_{g} \bar{\rho}_{a}^{2}}=\frac{2}{3} \bar{r}_{g}
$$

The general solution of the first equation in Equations (56) is [12] 


$$
\left(\frac{1}{3} \bar{\rho}_{e}^{2}+\frac{5}{12} \bar{r}_{g} \bar{\rho}_{e}+\frac{5}{8} \bar{r}_{g}^{2}\right) \sqrt{\bar{\rho}_{e}\left(\bar{\rho}_{e}-\bar{r}_{g}\right)}+\frac{5}{8} \bar{r}_{g}^{3} \ln \left(\sqrt{\bar{\rho}_{e}}+\sqrt{\bar{\rho}_{e}-\bar{r}_{g}}\right)=\frac{1}{3} \bar{r}^{3}+C_{5}
$$

The integration constant can be found from the boundary condition on the so here surface according to which $\bar{\rho}_{e}(\bar{r}=1)=\bar{\rho}_{a}$. Then,

$$
C_{5}=\left(\frac{1}{3} \bar{\rho}_{a}^{2}+\frac{5}{12} \bar{r}_{g} \bar{\rho}_{a}+\frac{5}{8} \bar{r}_{g}^{2}\right) \sqrt{\bar{\rho}_{a}\left(\bar{\rho}_{a}-\bar{r}_{g}\right)}+\frac{5}{8} \bar{r}_{g}^{3} \ln \left(\sqrt{\bar{\rho}_{a}}+\sqrt{\bar{\rho}_{a}-\bar{r}_{g}}\right)-\frac{1}{3}
$$

Thus, the functions $\rho_{i}(r)$ and $\rho_{e}(r)$ are specified by Equations (58) and (61). The first boundary condition in Equations (28) according to which $\rho_{i}(a)=\rho_{e}(a)=\rho_{a}$ is satisfied which follows from Equations (60) and (62). The second boundary condition in Equations (28) according to which $g_{i}(a)=g_{e}(a)$ is satisfied because the obtained solution is based on the condition $g_{i} \rho_{i}^{2}=g_{e} \rho_{e}^{2}$ from which it follows that if the function $\rho(r)$ is continuous at $r=a$, the function $g(r)$ is also continuous. For low levels of gravitation, i.e. for $\bar{r}_{g} \ll 1$, Equations (58) and (61) yield $\rho_{i}=\rho_{e}=r$.

Consider Equations (60) and (62) which allow us to satisfy the boundary conditions, i.e., to solve the problem that turned out to be critical for the solutions discussed above. As follows from Equation (62), the solution exists if $\bar{\rho}_{a} \geq \bar{r}_{g}$. Otherwise, the solution becomes imaginary. The minimum possible value of $\bar{\rho}_{a}$ is $\bar{r}_{g}$. Assume that this minimum value corresponds to the sphere radius $a_{g}$. Then, substituting $\bar{\rho}_{a}=\rho_{a} / a_{g}=r_{g} / a_{g}$ in Equation (60), we get

$$
\sqrt{\frac{a_{g}}{r_{g}}} \sin ^{-1}\left(\frac{r_{g}}{a_{g}} \sqrt{\frac{r_{g}}{a_{g}}}\right)-\frac{r_{g}}{a_{g}} \sqrt{1-\left(\frac{r_{g}}{a_{g}}\right)^{3}}=\frac{2 r_{g}}{3 a_{g}}
$$

The solution of Equation (63) is $a_{g}=1.115 r_{g}$. Thus, the critical radius is larger than the gravitational radius. For $a=a_{g}$, the solution is not singular and gives finite values for the metric coefficients. Particularly, for $\rho=\rho_{a}$ we get $g_{e}=g_{i}=1.243$ and $\rho_{e}=\rho_{i}=0.8968 a$. For $a<a_{g}$, the solution becomes imaginary which means that GRT is not valid for such high level of gravitation. For the sphere with radius $a_{g}$, the escape velocity is equal to the velocity of light and such sphere is invisible [18]. More results concerning the discussed solution can be found elsewhere [12].

\section{Conclusion}

As follows from the foregoing analysis, the Schwarzschild solution after some minor correction and reconstruction coincides with the traditional [3] interpretation of this solution. Both solutions do not satisfy the boundary condition on the fluid sphere surface for the radial space metric coefficient. A proposed model of the Riemannian space as the Euclidean space of variable density allows us to obtain the solution which satisfies equations GRT and all boundary conditions for the spherically symmetric problem for a fluid sphere. In future, the authors plan to generalize the approach discussed in Section 4 to the axially symmetric problem of GRT. 


\section{Conflicts of Interest}

The authors declare no conflicts of interest regarding the publication of this paper.

\section{References}

[1] Schwarzschild, K. (1916) Sitz. Preuss. Akad. Wiss., 1916, 189-207.

[2] Schwarzschild, K. (1916) Sitz. Preuss. Akad. Wiss., 1916, 424-432.

[3] Synge, J.L. (1960) Relativity: the General Theory. Amsterdam, North Holland.

[4] Weinberg, S. (1972) Gravitation and Cosmology. Amsterdam, North Holland.

[5] Misner, S.W., Thorne, K.S. and Wheeler, G.A. (1973) Gravitation. W.H. Freeman and Co., San Francisco.

[6] Chandrasecar, S. (1983) The Mathematical Theory of Black Holes. Oxford University Press, Oxford.

[7] Frolov, V.P. and Zelnikov, A. (2011) Introduction to Black Hole Physics. Oxford University Press, Oxford. https://doi.org/10.1093/acprof:oso/9780199692293.001.0001

[8] Logunov, A.A., Mestvirishvili, M.A. and Petrov, V.A. (2004) Physics-Uspekhi, 47, 607-621. (In Russian) https://doi.org/10.1070/PU2004v047n06ABEH001817

[9] Fock, V. (1959) The Theory of Space, Time and Gravitation. Pergamon Press, London.

[10] Landau, L.D. and Lifshits, E.M. (1988) Field Theory. Nauka, Moscow. (In Russian)

[11] Vasiliev, V.V. and Fedorov, L.V. (2017) Applied Physics Research, 6, 8-13. https://doi.org/10.5539/apr.v9n6p8

[12] Vasiliev, V.V. (2017) Journal of Modern Physics, 8, 1087-1100. https://doi.org/10.4236/jmp.2017.87070

[13] Einstein, A. (1916) Annalen der Physik, 49, 769-819. https://doi.org/10.1002/andp.19163540702

[14] Einstein, A. (1939) Annals of Mathematics, 40, 922-936. https://doi.org/10.2307/1968902

[15] Vasiliev, V.V. and Fedorov, L.V. (2012) Applied Physics Research, 2, 166-174.

[16] Vasiliev, V.V. (1989) Mechanics of Solids, 5, 30-34.

[17] Vasiliev, V.V. and Fedorov, L.V. (2013) Applied Physics Research, 1, 115-125.

[18] Vasiliev, V.V. and Fedorov, L.V. (2015) Applied Physics Research, 3, 84-92. 